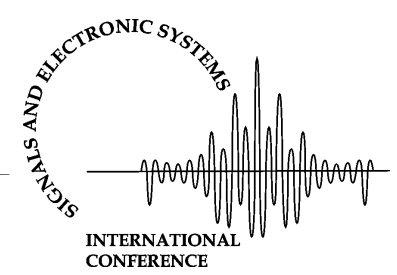

\title{
Short periodic orbits for the Lorenz system
}

\author{
Zbigniew Galias \\ Department of Electrical Engineering \\ AGH University of Science and Technology \\ al. Mickiewicza 30, 30-059 Kraków, Poland \\ Email: galias@agh.edu.pl
}

\author{
Warwick Tucker \\ Department of Mathematics \\ University of Bergen \\ Johannes Brunsgate 12, 5008 Bergen, Norway \\ Email: warwick.tucker@math.uib.no
}

\begin{abstract}
The existence of short periodic orbits for the Lorenz system is studied rigorously. We describe a method for finding all short cycles embedded in the chaotic attractor. We use the method of close returns to find initial points for the Newton operator, combined with interval tools for proving the existence of periodic orbits in a neighborhood of a pseudo-periodic orbit. All periodic orbits with period $p \leq 8$ of the Poincaré map for the Lorenz system are found.
\end{abstract}

\section{INTRODUCTION}

Despite the enormous progress in the theory of Dynamical Systems, proving the existence and location of periodic orbits for nonlinear continuous-time systems still is a highly nontrivial task. Even for planar polynomial systems (addressed in Hilbert's 16:th problem), this remains a huge challenge.

With the advent of computer-aided proofs, based on setvalued mathematics, several methods for just this task have been introduced. An important class of such methods use interval operators applied to a Poincaré map associated with the continuous system considered. When these methods are combined with approximations of periodic orbits, they can be used to extract many true cycles embedded in chaotic attractors.

Finding all short periodic orbits is even more challenging. It is known that this problem can be successfully solved for a certain class of systems including the Rössler system [1]. In this work we extend this method to a class of systems whose attractors (like the Lorenz attractor) contain an unstable equilibrium. For such systems there exist trajectories for which the return time is arbitrarily long. It is impossible to evaluate an interval flow-operator over a box containing such trajectories. For nearby boxes the evaluation procedure often fails or produces unusable results due to huge overestimation.

The Lorenz system [2] is well understood in terms of geometric models [3]. It has been shown to be chaotic in the topological sense for the case of non-classical [4] and classical [5] parameter values. The existence of a strange attractor for this system has been proved in [6].

In [7], we found the six shortest periodic orbits for the Lorenz system. This result was obtained by generating a graph representation of the dynamics of the Poincaré map, finding all short cycles in the graph, and verifying which cycles correspond to periodic orbits of the dynamical system. For longer periods the method fails due to long computation time, and the necessity of introducing many intermediate sections.

In this work, we use special properties of the Lorenz attractor which, combined with the interval tools, allow us to find all periodic orbits of much longer length.

\section{INTERVAL METHODS FOR FINDING PERIODIC ORBITS}

In this section, we briefly describe a general method which can be used to find all short periodic orbits. The first step of the method is a reduction of the continuous-time system to a discrete system using the concept of the Poincaré map.

\section{A. Poincaré map}

Let us choose hyperplanes $\Sigma_{1}, \Sigma_{2}, \ldots, \Sigma_{m}$ transversal to the flow, and let $\Sigma=\Sigma_{1} \cup \cdots \cup \Sigma_{m}$. The Poincaré map $P: \Sigma \mapsto \Sigma$ is defined as

$$
P(x)=\varphi(\tau(x), x),
$$

where $\tau(x)$ is the return time after which the trajectory $\varphi(t, x)$ returns to $\Sigma$. Periodic points of $P$ correspond to periodic orbits of the continuous system.

In order to study the existence of period- $p$ orbits of $P$ we construct the map $F$ defined by

$$
[F(z)]_{k}=x_{(k \bmod n)+1}-P\left(x_{k}\right), \quad k=1, \ldots, p,
$$

where $z=\left(x_{1}, \ldots, x_{p}\right)^{\mathrm{T}}$. Zeros of $F$ correspond to period $-p$ orbits of $P$, i.e., $F(z)=0$ if and only if $P^{p}\left(x_{1}\right)=x_{1}$.

\section{B. Interval methods}

Interval methods provide simple computational tests for uniqueness, existence, and nonexistence of zeros of a map within a given interval vector. In order to investigate the existence of zeros of $F$ in the interval vector $\mathbf{z}$, one evaluates an interval operator over $\mathbf{z}$. In this work we use the Krawczyk operator [8] defined by

$$
\mathrm{K}(\mathbf{z})=\hat{z}-C F(\hat{z})-\left(C F^{\prime}(\mathbf{z})-I\right)(\mathbf{z}-\hat{z}),
$$


where $\hat{z} \in \mathbf{z}$ and $C$ is an invertible matrix. In our implementation, we choose $\hat{z}$ to be the center of $\mathbf{z}$, and the preconditioning matrix $C$ to be the inverse of $F^{\prime}(\hat{z})$.

If $K(\mathbf{z})$ is enclosed in the interior of $\mathbf{z}$, i.e., $K(\mathbf{z}) \subset \operatorname{int} \mathbf{z}$, then $F$ has exactly one zero in $\mathbf{z}$. This property allows us to prove the existence and uniqueness of zeros.

In order to evaluate the interval operator for the map $F$ defined by (2), we need a method to find an enclosure of $P(\mathbf{x})$ and an enclosure of the Jacobian $P^{\prime}(\mathbf{x})$, where $\mathbf{x} \in \Sigma$ is an interval vector. These enclosures are found in interval arithmetic by rigorous integration of the differential equation and its variational equation. For details see [1].

\section{Searching for periodic orbits}

Now, we describe the procedure for detecting periodic orbits in chaotic systems. First, periodic orbits are extracted using the method of close returns [9]. We monitor a trajectory and look for parts of the trajectory coming close to the initial point. Then using the standard (non-interval) Newton method we sharpen the approximation, obtaining a quasi-periodic trajectory $z=\left(x_{1}, x_{2}, \ldots, x_{p}\right)$ of the Poincaré map. We create an interval vector $\mathbf{z}$ centered at the approximate position $z$ of the orbit, with the same diameter at all points along the orbit. Finally we check whether the image of $\mathbf{z}$ under the Krawczyk operator is enclosed in $\mathbf{z}$. If this condition holds, the existence (and local uniqueness) of a periodic orbit is proved. In the opposite case, we modify the interval vector and repeat the computations.

\section{SHORT PERIODIC ORBITS FOR THE LORENZ SYSTEM}

\section{A. Lorenz system}

The Lorenz system is described by the following set of equations

$$
\begin{aligned}
& \dot{x}_{1}=s x_{2}-s x_{1}, \\
& \dot{x}_{2}=r x_{1}-x_{2}-x_{1} x_{3}, \\
& \dot{x}_{3}=x_{1} x_{2}-q x_{3} .
\end{aligned}
$$

We consider the Lorenz system with the classical parameter values: $s=10, r=28, q=8 / 3$.

In what follows, we will use the diagonal form of the Lorenz system (it can be obtained using a linear change of variables), where the invariant manifolds of the origin are tangent to the coordinate axes:

$$
\begin{aligned}
& \dot{x}_{1}=\lambda_{1} x_{1}-k_{1}\left(x_{1}+x_{2}\right) x_{3}, \\
& \dot{x}_{2}=\lambda_{2} x_{2}+k_{1}\left(x_{1}+x_{2}\right) x_{3}, \\
& \dot{x}_{3}=\lambda_{3} x_{3}+\left(x_{1}+x_{2}\right)\left(k_{2} x_{1}+k_{3} x_{2}\right) .
\end{aligned}
$$

The constants are given by

$$
\begin{aligned}
& u=\sqrt{(s+1)^{2}+4 s(r-1)}, \\
& k_{1}=s / u \approx 0.2886, \quad k_{2}=(s-1+u) /(2 s) \approx 2.1828, \\
& k_{3}=(s-1-u) /(2 s) \approx-1.2828, \\
& \lambda_{1}=(-s-1+u) / 2 \approx 11.8277, \\
& \lambda_{2}=(-s-1-u) / 2 \approx-22.8277, \\
& \lambda_{3}=-q \approx-2.6667 .
\end{aligned}
$$

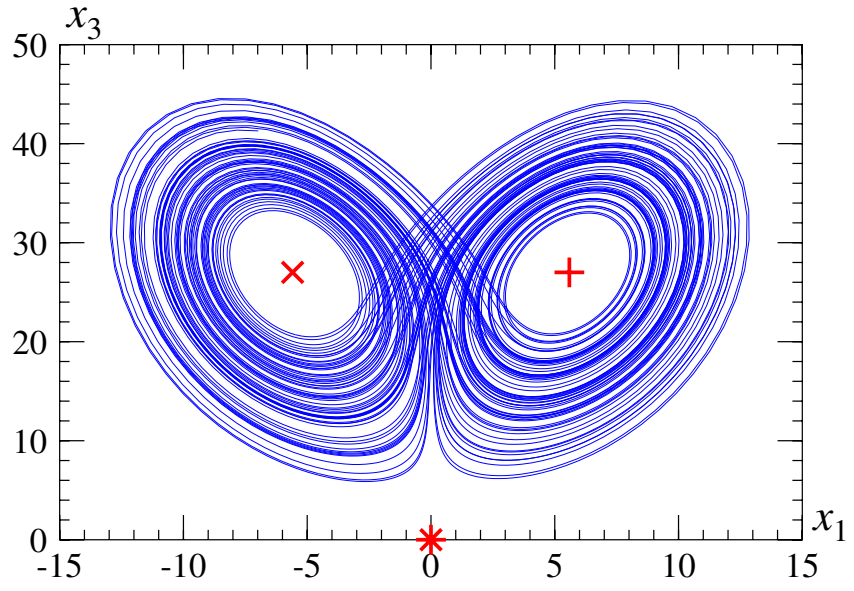

Fig. 1. A trajectory of the Lorenz system

From now on we will refer to (5) as the Lorenz system. A trajectory of the Lorenz system is shown in Fig. 1.

For the parameter values we consider, the Lorenz system has three equilibria. One of them is the origin $P_{0}=(0,0,0)^{T}$. It has one positive eigenvalue $\lambda_{1}$ and two negative eigenvalues $\lambda_{2}$ and $\lambda_{3}$ defined in (6). The coordinates of the other two equilibria, shown in Fig. 1 using symbols $\times$ and + , are

$$
P_{ \pm}=\left(\mp \frac{\lambda_{2}}{u} \sqrt{q(r-1)}, \pm \frac{\lambda_{1}}{u} \sqrt{q(r-1)}, r-1\right) .
$$

$P_{ \pm} \approx( \pm 5.589, \pm 2.896,27)$ have a pair of complex eigenvalues with positive real parts $\mu_{1,2} \approx 0.094 \pm j 10.19$, and one real negative eigenvalue $\mu_{3} \approx-13.854$.

\section{B. Trapping region and average return time}

Let us choose the Poincaré map $P$ defined by the section $\Sigma=\left\{x=\left(x_{1}, x_{2}, x_{3}\right): x_{3}=r-1, \dot{x}_{3}<0\right\}$.

We begin our analysis of the existence of periodic orbits in the Lorenz attractor by finding a trapping region containing the attractor of $P$. Observe that this cannot be done by a direct integration of the differential equation seeing that there are trajectories in the Lorenz attractor which pass arbitrarily close to the origin, and for which the return time is arbitrarily long. The trapping region is found using a modified Euler method with rigorous error bounds. In order to reduce expansion along the trajectories we use partitioning. When a box has expanded enough, it is partitioned, and the sub-boxes are then treated separately. This helps reduce the problems associated with the wrapping effect. Trajectories passing close to the fixed point at the origin are treated differently. We define a cube around the origin, and interrupt computations if the trajectory hits the cube. We then change to some carefully constructed normal form coordinates, and explicitly compute the exit of the trajectory. There are two different ways in which a rectangle can pass through the cube. If the box intersects the stable manifold of the origin, it is split along the line of intersection, and exits the cube in two pieces. Otherwise, the box flows out in one piece. After leaving the cube, we switch back to the original coordinates, and resume the numeric computations. For details see [6]. 
TABLE I

LOWER BOUND $t_{n}$ OF RETURN TIMES FOR $P^{n}$

\begin{tabular}{|r|r|l|}
\hline$n$ & $t_{n}$ & $t_{n} / n$ \\
\hline 1 & 0.537044 & 0.537044 \\
2 & 1.147434 & 0.573717 \\
5 & 2.981325 & 0.596265 \\
10 & 6.047894 & 0.6047894 \\
100 & 63.721019 & 0.63721019 \\
10000 & 6397.362682 & 0.6397362682 \\
\hline
\end{tabular}

The trapping region found using the method described above is composed of 14518 boxes of size $1 / 2^{7} \times 1 / 2^{7}$ (see Fig. 2). There are 514126 nonforbidden transition between the boxes.

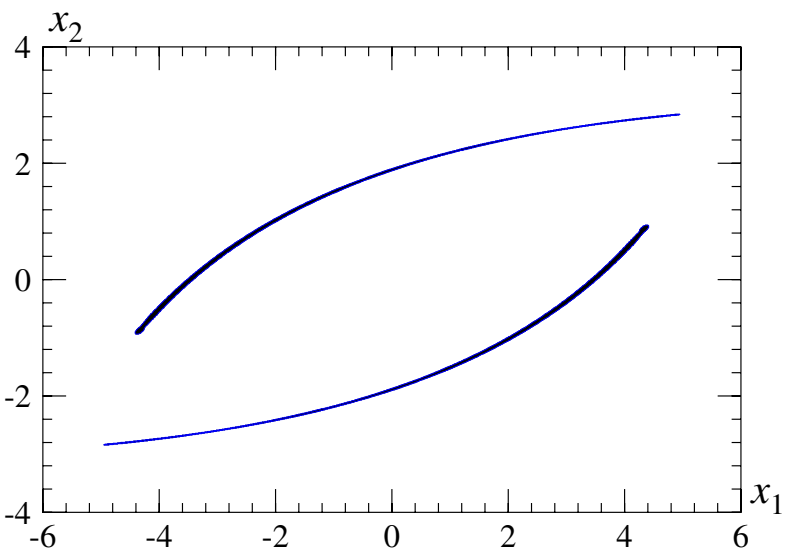

Fig. 2. Trapping region for the Poincaré map, $x_{3}=27$

Using information on return times for individual boxes, we can find bounds for the return time of the $n$th iterate of $P$. Since for some boxes an upper bound for the return time is infinite, the return time for the whole attractor is unbounded. Lower bounds of return times are collected in Table I.

It is clear that the average return time $\tau_{\text {aver }}$ between two crossings is larger than $t_{n} / n$ for each $n$. For $n=10000$ we obtain:

$$
\tau_{\text {aver }}>0.6397 \text {. }
$$

It follows that the length of any periodic orbit corresponding to a period $-n$ cycle of $P$ is larger than $n \cdot 0.6397$.

\section{Periodic orbits}

Let $\gamma$ denote the first intersection of the (two-dimensional) stable manifold of the origin with the return plane $\Sigma$. We will label each trajectory in the following way: if the trajectory intersects $\Sigma$ to the left of $\gamma$, then the intersection point is labelled with L, otherwise it is labelled with $\mathrm{R}$. In order to study periodic orbits we consider periodic symbolic sequences $s=\left(s_{1}, s_{2}, \ldots, s_{p}\right)$, where $s_{k}=\mathrm{L}$ or $s_{k}=\mathrm{R}$, for $k=1,2, \ldots p$. In [6], it was established that the Poincaré map induces a stable foliation of the forward invariant part of $\Sigma$. From this, it follows that a periodic symbolic sequence corresponds to at most one periodic orbit. Non-rigorous computations indicate that each periodic symbol sequence with no more
TABLE II

SHORT PERIODIC ORBITS, PERIOD $p$ OF THE ASSOCIATED PERIODIC ORBIT OF $P$, AND LENGTH $t$

\begin{tabular}{|c|c|c|l|}
\hline$p$ & $n$ & $t$ & $s$ \\
\hline 2 & 1 & {$[1.55864,1.55867]$} & LR \\
3 & 2,3 & {$[2.30589,2.30591]$} & LLR \\
4 & 4,5 & {$[3.02357,3.02360]$} & LLLR \\
4 & 6 & {$[3.08426,3.08429]$} & LLRR \\
5 & 7,8 & {$[3.72562,3.72566]$} & LLLLR \\
5 & 9,10 & {$[3.82024,3.82026]$} & LLLRR \\
5 & 11,12 & {$[3.86952,3.86956]$} & LLRLR \\
6 & 13,14 & {$[4.41774,4.41779]$} & LLLLLR \\
6 & 15,16 & {$[4.53409,4.53413]$} & LLLLRR \\
6 & 17 & {$[4.56629,4.56633]$} & LLLRRR \\
6 & 18,19 & {$[4.59379,4.59384]$} & LLLRLR \\
6 & 20,21 & {$[4.63712,4.63716]$} & LLRLRR \\
7 & 22,23 & {$[5.10301,5.10307]$} & \\
7 & 24,25 & {$[5.23417,5.23422]$} & \\
7 & 26,27 & {$[5.28632,5.28636]$} & \\
7 & 28,29 & {$[5.30118,5.30122]$} & \\
7 & 30,31 & {$[5.33089,5.33093]$} & \\
7 & 32,33 & {$[5.36986,5.36990]$} & \\
7 & 34,35 & {$[5.37050,5.37055]$} & \\
7 & 36,37 & {$[5.39419,5.39424]$} & \\
7 & 38,39 & {$[5.42910,5.42915]$} & \\
8 & 40,41 & {$[5.78337,5.78345]$} & \\
8 & 42,43 & {$[5.92496,5.92502]$} & \\
8 & 44,45 & {$[5.99042,5.99047]$} & \\
8 & 46,47 & {$[5.99729,5.99735]$} & \\
8 & 48 & {$[6.01000,6.01005]$} & \\
8 & 49,50 & {$[6.03521,6.03526]$} & \\
8 & 51,52 & {$[6.08233,6.08238]$} & \\
8 & 53,54 & {$[6.08380,6.08385]$} & \\
8 & 55,56 & {$[6.10803,6.10808]$} & \\
8 & 57,58 & {$[6.12143,6.12148]$} & \\
8 & 59,60 & {$[6.12231,6.12236]$} & \\
8 & 61,62 & {$[6.13510,6.13515]$} & \\
8 & 63,64 & {$[6.15469,6.15475]$} & \\
8 & 65,66 & {$[6.17585,6.17590]$} & \\
8 & 67 & {$[6.18749,6.18754]$} & \\
8 & 68,69 & {$[6.19458,6.19463]$} & \\
\hline & & & \\
\end{tabular}

than 24 repeating symbols corresponds to a periodic orbit (compare [10]).

There is no periodic orbit corresponding to a single letter symbol. This has been verified by checking that there are no period-1 cycles in the graph (see [7]).

All periodic orbits of $P$ with period $p \leq 8$ were found using the combination of the method of close returns and interval tools. In order to achieve this, we first generated a trajectory consisting of 200000 points of the Poincaré map $P_{2}$, defined by the section $\Sigma_{2}=\left\{x=\left(x_{1}, x_{2}, x_{3}\right): x_{3}=r-1\right\}$. The search was then limited to periodic orbits with period $p \leq 16$ (this corresponds to periodic orbits with period $p \leq 8$ for the map $P$. Next, we located quasi-periodic trajectories (returning to the neighborhood with radius 0.005 of the initial point). Each found quasi-periodic trajectory was used as an initial point for a Newton iteration to find periodic orbits in its neighborhood. Finally, we proved the existence of a nearby true periodic orbit using the Krawczyk operator. If the same periodic orbit had already been found it was skipped, otherwise its position was recorded.

The results are reported in Table II. For each orbit found, its period $p$, its position $n$ in the list of all periodic orbits 
(a) $p=2, n=1, \mathrm{LR}$

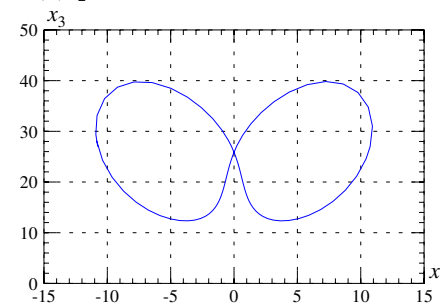

(e) $p=5, n=7$, LLLLR

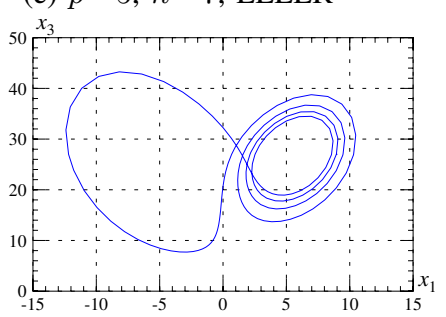

(i) $p=6, n=15$, LLLLRR

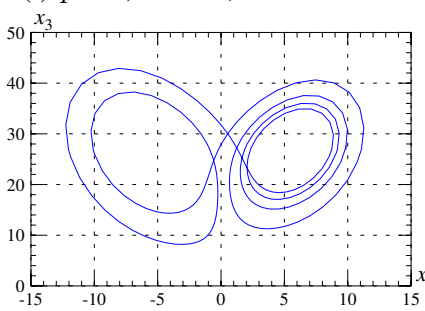

(b) $p=3, n=2$, LLR

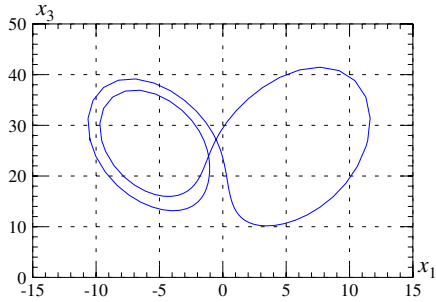

(f) $p=5, n=9$, LLLRR

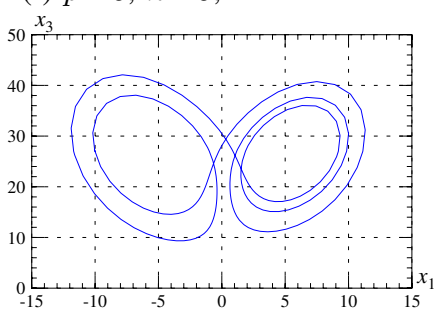

(j) $p=6, n=17$, LLLRRR

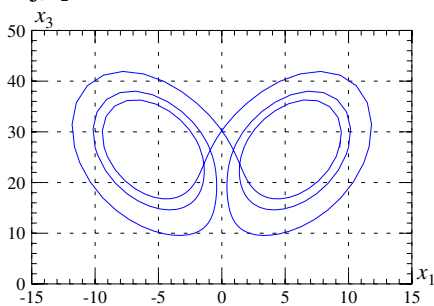

(c) $p=4, n=4$, LLLR

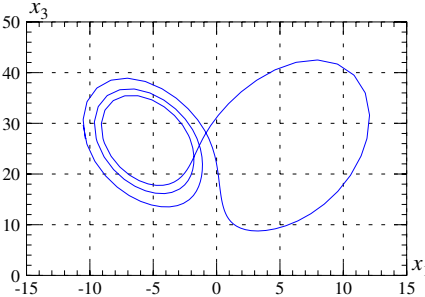

(g) $p=5, n=11$, LLRLR

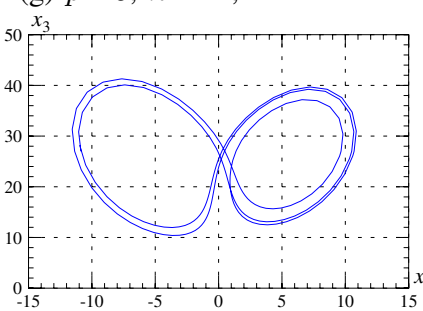

(k) $p=6, n=18$, LLLRLR

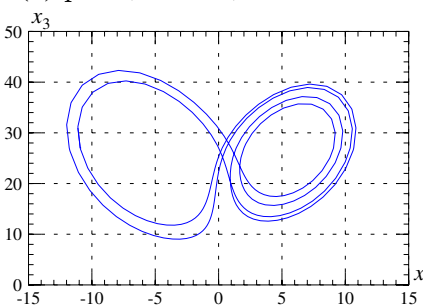

(d) $p=4, n=6$, LLRR

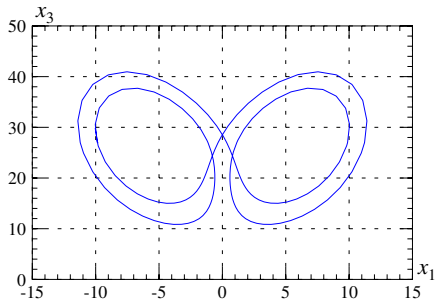

(h) $p=6, n=13$, LLLLLR

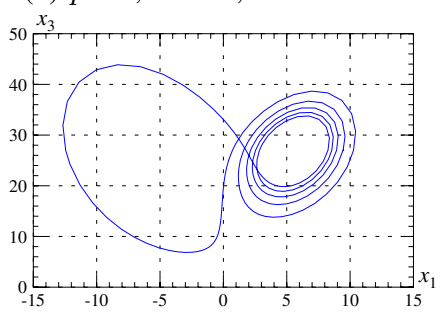

(g) $p=6, n=20$, LLRLRR

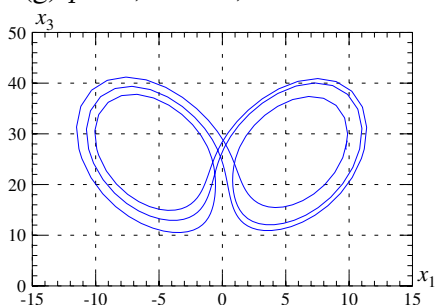

Fig. 3. Short periodic orbits

sorted by flow-times, the interval containing the flow-time, and for shorter orbits, its corresponding symbol sequence are given. There are 5 self symmetric orbits $(n=1,6,17,47,67)$, one for each period $p=2,4,6$, and two for period 8 . These are periodic orbits with symmetric symbol sequences: $L R$, $L L R R, L L L R R R, L L L L R R R R, L L R L R R L R$. There are also 32 pairs of orbits symmetric to each other. Clearly, they have equal flow-times. For each pair, we report only the one with the number of L's not larger than the number of R's. This gives the total number of 69 orbits. Periodic orbits with period $p \leq 6$ are presented in Fig. 3. For each pair of symmetric orbits, only one orbit is plotted.

Let us note that there are exactly 69 periodic symbolic sequences with no more than 8 repeating symbols, and recall that each periodic symbol sequence corresponds to at most one periodic orbit. Hence, we have confirmed that there are exactly 69 periodic orbits of $P$ with period $p \leq 8$ (compare [10]). The flow-time of any other periodic orbit of $P$ is larger than $9 \cdot 0.6397=5.7573($ compare Eq. $(7))$. Since the first 39 periodic orbits have periods shorter than 5.43 , it follows that these periodic orbits are the shortest. Note that it is possible that an orbit with period $p=9$ has a flow-time shorter than that of some period -8 orbits.

\section{CONCLUSION}

Using the search method for short periodic orbits and a theoretical argument for the Lorenz system, we have found all periodic orbits with period $p \leq 8$ of the Poincare map associated with the Lorenz system.

\section{ACKNOWLEDGMENT}

This work was supported in part by the AGH University of Science and Technology, grant no. 10.10.120.133.

\section{REFERENCES}

[1] Z. Galias, "Counting low-period cycles for flows," Int. J. Bifurcation and Chaos, vol. 16, no. 10, pp. 2873-2886, 2006.

[2] E. Lorenz, "Deterministic non-periodic flow," Journal of Atmospheric Science, vol. 20, pp. 130-141, 1963.

[3] C. Sparrow, The Lorenz equations: Bifurcations, Chaos, and Strange Attractors. New York: Springer Verlag, 1982.

[4] K. Mischaikow and M. Mrozek, "Chaos in the Lorenz equations: a computer assisted proof," Bull. Amer. Math. Soc., vol. 32, no. 1, pp. 66-72, 1995.

[5] Z. Galias and P. Zgliczyński, "Computer assisted proof of chaos in the Lorenz equations," Physica D, vol. 115, pp. 165-188, 1998.

[6] W. Tucker, "The Lorenz attractor exists," C. R. Acad. Sci. Paris, vol. 328, pp. 1197-1202, 1999.

[7] Z. Galias and W. Tucker, "Rigorous study of short periodic orbits for the Lorenz system," in Proc. IEEE Int. Symposium on Circuits and Systems, ISCAS'08, Seattle, May 2008, pp. 764-767.

[8] A. Neumaier, Interval methods for systems of equations. Cambridge University Press, 1990.

[9] D. Lathrop and E. Kostelich, "Characterisation of an experimental strange attractor by periodic orbits," Phys. Rev. A, vol. 40, no. 7, pp. 4028-4031, 1989.

[10] D. Viswanath, "Symbolic dynamics and periodic orbits of the Lorenz attractor," Nonlinearity, vol. 16, pp. 1035-1056, 2003. 